\title{
Effect of Electrical and Electromechanical Stimulation on PC12 Cell Proliferation and Axon Outgrowth
}

OPEN ACCESS

Edited by:

Kristen L. Billiar,

Worcester Polytechnic Institute,

United States

Reviewed by:

Farnaz Ghorbani,

University of Erlangen Nuremberg,

Germany

Sanjeev Kumar Mahto, Indian Institute of Technology (BHU),

India

${ }^{*}$ Correspondence:

Jin Qian

jqian@zju.edu.cn

Jie Sun

nbyysj@sina.com

${ }^{+}$These authors have contributed equally to this work

Specialty section: This article was submitted to

Biomechanics,

a section of the journal Frontiers in Bioengineering and

Biotechnology

Received: 13 August 2021

Accepted: 15 September 2021

Published: 21 October 2021

Citation:

Xu K, Liu X, Li X, Yin J, Wei P, Qian J and Sun $J$ (2021) Effect of Electrical and Electromechanical Stimulation on PC12 Cell Proliferation and Axon Outgrowth.

Front. Bioeng. Biotechnol. 9:757906.

doi: 10.3389/fbioe.2021.757906
Kailei Xu ${ }^{1,2,3+}$, Xixia $\mathrm{Liu}^{2,3,4 t}$, Xiaokeng $\mathrm{Li}^{2,3}$, Jun Yin ${ }^{2,3}$, Peng Wei ${ }^{5}$, Jin $\mathrm{Qian}^{6 *}$ and Jie Sun ${ }^{1,7 *}$

${ }^{1}$ Central Laboratory, Ningbo First Hospital, Ningbo, China, ${ }^{2}$ The State Key Laboratory of Fluid Power and Mechatronic Systems, School of Mechanical Engineering, Zhejiang University, Hangzhou, China, ${ }^{3}$ Key Laboratory of 3D Printing Process and Equipment of Zhejiang Province, School of Mechanical Engineering, Zhejiang University, Hangzhou, China, ${ }^{4}$ School of Mechanical Engineering, Guizhou University, Guiyang, China, ${ }^{5}$ Department of Hand and Foot Microsurgery, Ningbo First Hospital, Ningbo, China, ${ }^{6}$ Key Laboratory of Soft Machines and Smart Devices of Zhejiang Province, Department of Engineering Mechanics,

Zhejiang University, Hangzhou, China, 'Department of Neurosurgery, Ningbo First Hospital, Ningbo, China

Peripheral nerve injuries have become a common clinical disease with poor prognosis and complicated treatments. The development of tissue engineering pointed a promising direction to produce nerve conduits for nerve regeneration. Electrical and mechanical stimulations have been incorporated with tissue engineering, since such external stimulations could promote nerve cell proliferation, migration and differentiation. However, the combination of electrical and mechanical stimulations (electromechanical stimulation) and its effects on neuron proliferation and axon outgrowth have been rarely investigated. Herein, silver nanowires (AgNWs) embedded polydimethylsiloxane (PDMS) electrodes were developed to study the effects of electromechanical stimulation on rat pheochromocytoma cells (PC12 cells) behaviors. AgNWs/PDMS electrodes demonstrated good biocompatibility and established a stable electric field during mechanical stretching. PC12 cells showed enhanced proliferation rate and axon outgrowth under electrical stimulation alone, and the cell number significantly increased with higher electrical stimulation intensity. The involvement of mechanical stretching in electrical stimulation reduced the cell proliferation rate and axon outgrowth, compared with the case of electrical stimulation alone. Interestingly, the cellular axons outgrowth was found to depend on the stretching direction, where the axons prefer to align perpendicularly to the stretch direction. These results suggested that AgNWs/PDMS electrodes provide an in vitro platform to investigate the effects of electromechanical stimulation on nerve cell behaviors and can be potentially used for nerve regeneration in the future.

Keywords: nerve regeneration, electrical field, mechanical stretch, silver nanowires, nerve cells, PDMS 


\section{INTRODUCTION}

The nervous system, composed of the central nervous system and the peripheral nervous system, is a vast conductive network that coordinates actions and sensory information of the human body through the transmission of electrical signals to and from different tissues. The central nervous system includes the brain and the spinal cord; while the peripheral nervous system is composed of numerous long fibers or axons, which connect with the central nervous system. Peripheral nervous injuries (PNI) have become a common clinical disease, with millions of cases happening annually around the world, and more than 200,000 repair surgeries are performed in the US every year (Du et al., 2018). The PNI generally causes a poor prognosis due to the complicated treatments and high morbidity. About $60 \%$ of PNI patients have poor rehabilitation of exercise abilities, and some of them require long-term care by others, which significantly affects patients' life quality (Gu et al., 2011; Wieringa et al., 2018). Therefore, it is of great importance to develop effective PNI repair strategies.

Clinical treatments for PNI have not changed much in the past decades, mainly including end-to-end and fascicular suture repair techniques, grafting sensory nerves, and autografts as the gold standard. However, the autografts still have many limitations, like donor repeated operations, nerve unavailability, local tissue adhesion, and size mismatch (Daly et al., 2013; Tang et al., 2013; Jahromi et al., 2019). Recently, the development of tissue engineering has pointed a new direction on PNI treatment, i.e., nerve conduit (Suo et al., 2018; Liu et al., 2021; Wang et al., 2021), which is an artificial hollow tube as an alternative that connects the two ends of the injured nerve to support nerve migration and regeneration.

Successful nerve conduit needs to provide not only physical support for growing neurites (Yin et al., 2017; Zhang et al., 2020), but also biological signals to promote nerve cell migration, proliferation, axon outgrowth, and orientation (Huang and Huang, 2006). These behaviors of nerve cells in tissue engineering could be influenced by many cues of substrate surface topographies, like aligned fibers, roughness, and pores, and external stimulations (Fozdar et al., 2010; Hoffman-Kim et al., 2010; Clarke et al., 2011). Electrical stimulation has been shown to promote neurite outgrowth, axon regeneration, and neural repair in vitro and in vivo (Kotwal and Schmidt, 2001; Bhang et al., 2012; Du et al., 2016), which might be due to its promotion effect on nerve cell adhesion, growth, migration, and proliferation. Micropatterned silk protein films sputter-coated with metal electrodes have been shown to promote the nerve cell axon outgrowth under electrical stimulation (Hronik-Tupaj et al., 2013). Animal studies also demonstrated that electrical stimulation can accelerate the maturity of regenerated nerves (Lu et al., 2008). Both peripheral nerve fibers and cells are constantly subjected to stretching and compression forces during the movement of the limbs (Phillips et al., 2004); therefore, the PNI repair and nerve cell growth are also affected by mechanical stimulation (Ishibashi et al., 2016; Wu et al., 2019). The adhesion (Love et al., 2017), signaling pathways (Grove and Brophy, 2014), and gene expression (Gupta et al.,
2012) of nerve cells have been found to be tightly regulated by mechanical stimulations, including the change of tissue stiffness or shear stress. Although significant progress has been made in the application of electrical stimulation and mechanical stimulations for neural tissue engineering, the combination of these two factors was rarely investigated.

In this study, polydimethylsiloxane (PDMS) was combined with silver nanowires (AgNWs) to prepare AgNWs/PDMS electrodes that were used to study the responses of rats pheochromocytoma (PC12) cells to electrical stimulation as well as electromechanical stimulation. AgNW has been widely used for flexible/wearable electrodes or stretchable applications, due to its excellent electrical conductivity, high mechanical strength and good biocompatibility. The role of AgNWs in this study acted as a conductive electrode material to provide a stable electrical field to study the effect of electrical stimulation on PC12 cellular behaviors. PDMS was a commonly used substrate for implantable electrodes, due to its low water absorption and high flexibility and elasticity (Jeong et al., 2016). Our group has used PDMS as substrate to study the behavior of PC12 cells under mechanical stimulation alone previously (Lin et al., 2020). PC12 cells demonstrated axon orientation perpendicular to the stretch direction, while the cell proliferation was not influenced. The role of PDMS in this study is to prevent the direct contact between PC12 cells and AgNWs, and also provide sufficient mechanical strength and high elasticity to protect the AgNWs from breaking during electromechanical stimulation. The prepared AgNWs/PDMS electrodes were characterized with a scanning electron microscope (SEM) for the microscopic surface structure of the AgNWs layer, and the electromechanical properties of AgNWs/ PDMS electrodes were characterized by cyclic voltammetry during the cyclic tensile test using a universal resistance meter. In vitro cell culture was performed with PC12 cells on the electrodes for $120 \mathrm{~h}$ and analyzed with Calcein-AM for cell viability, fluorescence imaging for cell density counting, cell axon outgrowth and orientation measurement at 24,72, and $120 \mathrm{~h}$. These results demonstrated that the AgNWs/PDMS electrodes are compatible with nerve cell culture as an integrated electromechanical stimulator for cell proliferation and axon outgrowth.

\section{METHODS}

\section{Materials}

PDMS was purchased from Sylgard (United States). AgNWs (diameter $30-50 \mathrm{~nm}$, length $10-30 \mu \mathrm{m}, 10 \mathrm{mg} / \mathrm{ml}$ in DMF) were purchased from ECHUANG (China). Dulbecco's modified Eagle's medium (DMEM) was purchased from Hyclone (Logan, UT). Poly-L-lysine (PLL) was purchased from Beyotime (China). Fetal bovine serum (FBS) was purchased from Gibco (Waltham, MA). Penicillin/ streptomycin $(\mathrm{P} / \mathrm{S})$ was purchased from Invitrogen (Carlsbad, $\mathrm{CA}$, United States). Calcein-AM was purchased from Aladdin (China). The PC12 cell line was purchased from Cell Bank of the Chinese Academy of Science (Shanghai, China, Catalog \#: TCR9), 


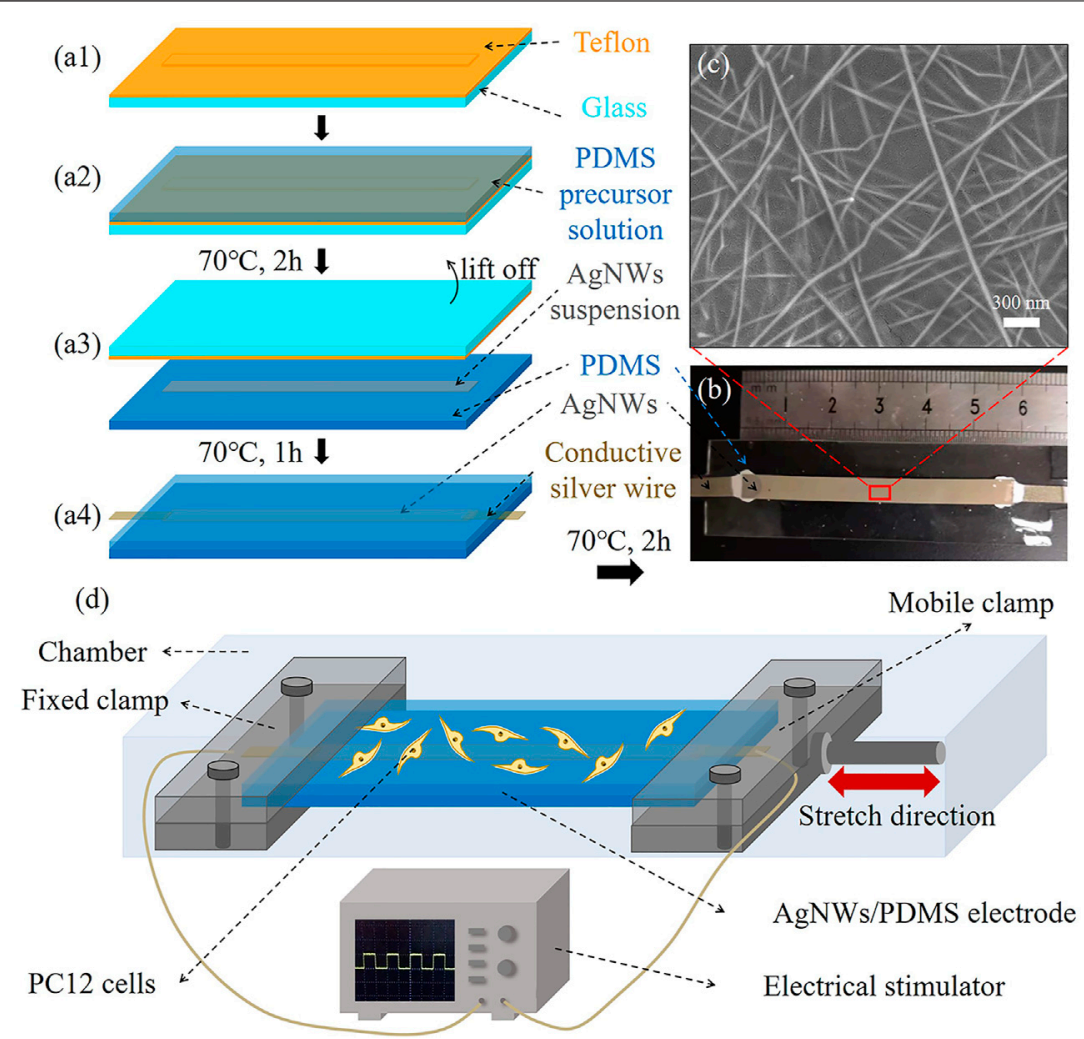

FIGURE 1 | (A) Fabrication procedure of the AgNWs/PDMS electrode preparation. (B) Photograph of the prepared AgNWs/PDMS electrode. (C) SEM image of the surface of the AgNWs layer in the AgNWs/PDMS electrode. (D) Schematic illustration of electromechanical stimulation.

which are highly differentiated already. PDMS is a commonly used elastomer due to its good biocompatibility and mechanical properties, and Ag-based materials have inherently high electrical conductivity (Han et al., 2019). PC12 cells are widely used to study neural behavior since they could differentiate into the nerve cells, which have neurite, dendrite, and axon, and form synapses with neighboring cells to build neural networks (Foley et al., 2005).

\section{Preparation of AgNWs/PDMS Electrode}

The process for the preparation of AgNWs/PDMS electrodes is shown in Figure 1A, including four procedural steps. First, a glass slide $(75 \mathrm{~mm} \times 25 \mathrm{~mm})$ was cleaned with $70 \%$ ethanol, blowdried, and attached with a Teflon mold that had a convex part [ $5 \mathrm{~mm} \times 70 \mathrm{~mm} \times 0.1 \mathrm{~mm}$, (Figure 1A)]. Second, the PDMS precursor solution was prepared by mixing pre-polymer gel and crosslinker at a ratio of 10:1 (w/w), then coated on the Teflon mold to form a thickness of $0.5 \mathrm{~mm}$ and solidified at $70^{\circ} \mathrm{C}$ for $2 \mathrm{~h}$ (Figure 1A). Third, the solidified PDMS was demoulded and inverted, filled with $150 \mu \mathrm{L}$ of AgNWs suspension into the rectangular groove $(5 \mathrm{~mm} \times 70 \mathrm{~mm} \times 0.1 \mathrm{~mm})$ that formed by the convex part of the Teflon mold, and dried at $70^{\circ} \mathrm{C}$ for $1 \mathrm{~h}$ to remove DMF (Figure 1A). In particular, the conductive silver wires were connected to the two ends of solidified AgNWs with conductive glue (Figure 1A). Finally, the PDMS substrate containing AgNWs and conductive wires were covered with
$0.5 \mathrm{~mm}$-thick PDMS precursor solution and then solidified at $70^{\circ} \mathrm{C}$ for $2 \mathrm{~h}$ to realize the encapsulation and obtain AgNWs/ PDMS electrodes (Figure 1B).

\section{SEM Imaging}

The surface morphology of the deposited AgNWs layer was observed by coating with a thin layer of gold using a scanning electron microscope (SU8010, Hitachi, Japan), where two PDMS layers of the electrodes were stripped and the PDMS base layer was removed.

\section{Electromechanical Properties of the AgNWs/PDMS Electrode}

Excellent stretchability and electrical stability in the stretched state are the basis of electrodes for biomedical applications. The electromechanical properties of the prepared AgNWs/PDMS electrodes were characterized by cyclic voltammetry. Briefly, the electrical resistance of the AgNWs/PDMS electrode was recorded under normal and stretch conditions using an electronic extensometer (UTM17290-220, Shenzhen SUNS, China) and a universal resistance meter (KEYSIGHT, 34465A, China). The AgNWs/PDMS electrode was clipped onto the extensometer with an initial effective stretching distance of $30 \mathrm{~mm}$. A periodic tensile test was applied to the sample with a crosshead speed of $1.25 \mathrm{~mm} / \mathrm{s}$. The electrode sample was 
elongated to reached $30 \%$ strain $(9 \mathrm{~mm})$ and then relaxed back to $0 \%$ strain as one stretching cycle. This was repeated 100 times, and the resistance was measured/recorded by the resistance meter at a frequency of $2 \mathrm{~Hz}$.

\section{Cell Culture on AgNWs/PDMS Electrode Under Electrical Stimulation}

PC12 cells were expanded in the Petri dishes until $70 \%$ confluency at $37^{\circ} \mathrm{C}$ and $5 \% \mathrm{CO}_{2}$ in a humidified incubator (Thermo Scientific, United States). The fully supplemented media for PC12 cell culture was DMEM with $10 \%$ FBS and $1 \%$ $\mathrm{P} / \mathrm{S}$. The cells were detached from the Petri dish with trypsinEDTA, centrifuged at 1000 RPM for $5 \mathrm{~min}$, and resuspended in fully supplemented media at a concentration of $8 \times$ $10^{4}$ cells/mL. The AgNWs/PDMS electrode was coated with PLL and sterilized with $75 \%$ ethanol. Then $5 \mathrm{ml}$ of cell suspension was added to the surface of the AgNWs/PDMS electrode and incubated at $37^{\circ} \mathrm{C}$ and $5 \% \mathrm{CO}_{2}$ in a humidified incubator. An electrical stimulator (Tektronix, United States) was connected to the AgNWs/PDMS electrode and provided a square wave voltage with different amplitude $(60 \mathrm{mV}$, $120 \mathrm{mV}, 240 \mathrm{mV}$ ) at a frequency of $20 \mathrm{~Hz}$. Cells were cultured for $120 \mathrm{~h}$ and stimulated for $4 \mathrm{~h}$ per day, which should not cause bio-demage. Similar electrical stimulation conditions were also performed in other studies. PC12 cells cultured on conducting polymer polypyrrole were stimulated with $720 \mathrm{mV}$ to investigate the extracellular matrix change (Kotwal and Schmidt, 2001). Schwann cells were electrically stimulated under $200 \mathrm{mV}$ continuously for a maximum of $48 \mathrm{~h}$ with little necrosis (Du et al., 2016).

\section{Electromechanical Stimulation on Cell Culture}

The AgNWs/PDMS electrode was seeded with $5 \mathrm{ml}$ of cell suspension and incubated for $4 \mathrm{~h}$ for cell attachment. Then the sample was transferred to a biological tensile tester (MechanoCulture T6, CellScale, Canada), exposed to cyclic tensile stretching $(10 \%$ strain at $0.25 \mathrm{~Hz})$ and electrical stimulation with a square wave voltage of $240 \mathrm{mV}$ and $20 \mathrm{~Hz}$. Cell culture without any stimulation was served as a control.

\section{Fluorescence Stain for Cell Proliferation and Axon Outgrowth Analysis}

PC12 cells were stained with Calcein-AM for cell morphology imaging. The electrode seeded with PC12 cells were washed with D-PBS and incubated with $1 \mu \mathrm{g} / \mathrm{ml}$ Calcein-AM for $30 \mathrm{~min}$ at $37^{\circ} \mathrm{C}$ and $5 \% \mathrm{CO}_{2}$ in an incubator. The sample was washed with D-PBS three times and imaged with a fluorescence microscope (Ti-S, Nikon, Japan). The axon outgrowth of PC12 cells was characterized by length (L) and the orientation $(\theta)$, where $\mathrm{L}$ is the end-to-end distance from soma to axon tip and $\theta$ is the angle between axon and electric current or stretch direction (Shineh et al., 2019; Krishnamoorthy et al., 2020).

\section{Statistical Analysis}

All quantitative data were analyzed and presented as the mean \pm standard deviation and the comparison between two means was analyzed using Tukey's tests, in which $p<0.05$ was considered statistical significance. For cell density measurement, more than 10 images were analyzed. For axon orientation and length, the distribution of orientation $\theta$ was divided into 12 uniform intervals with a span of $30^{\circ}$ (Lin et al., 2020). Each data of axon length and cell orientation was obtained based on the measurements $(n>150$ cells for each condition) using ImageJ (version 1.8.0, National Institutes of Health, United States).

\section{RESULTS}

\section{AgNWs/PDMS Electrode Characterization}

Representative AgNWs/PDMS electrode with a total length of $\sim 60 \mathrm{~mm}$ is shown in Figure 1B. It is noted that the pattern of electrodes (AgNWs) could be easily modified by changing the shape of the Teflon mold through 3D printing. The SEM image of the AgNWs surface after pilling off the PDMS substrate was shown in Figure 2C. Originally, the AgNW nanofibers were brittle and easy to fracture, however, the AgNW nanofibers in the prepared electrodes formed an interconnected network with PDMS penetration (Figure 2C) due to its flowability (viscosity: $5,467 \pm 370 \mathrm{mPas}$ ) and low surface energy of the encapsulation layer PDMS precursor solution, resulting in an excellent elasticity and tensile electrical stability of the electrodes.

Figure 2 showed the resistance variation of the electrodes during the cyclic tensile stretching process. The initial resistance of the electrodes was only $3.71 \Omega$, indicating that AgNWs/PDMS electrodes had good electrical conductivity. During the first stretch-relaxation cycle, the resistance increases to $38.81 \Omega$, which is almost 10 times higher than the initial value. After the strain was fully released, the resistance recovered back to $5.18 \Omega$, which was still $40 \%$ higher than the initial resistance. The resistance showed a major hysteresis during the first stretchrelaxation cycle, but essentially no hysteresis was found for the subsequent cycles (Figure 2A), indicating that the plastic deformation of the AgNWs during multiple loading cycles was mainly caused by the first stretch-relaxation cycle. The resistance increased from 5.18 to $41.94 \Omega$ during the second stretchrelaxation cycle, which was $8.1 \%$ higher than the first cycle, and it returned to $5.18 \Omega$ after the stretch was released. In the third stretch-relaxation cycle, the electrode resistance increased from 5.18 to $43.06 \Omega$. The resistance value increased by $2.7 \%$ compared to the second cycle, indicating that the maximum resistance of the electrode increased with the increase of stretch times during the first three cycles. Due to the variation of electrode resistance, the equivalent current also varies from 16 to $64 \mathrm{~mA}$. While the PC12 cells were cultured in an electrical field rather than in contact with AgNWs directly, the cell viability could be ensured.

To explore the changes of electrode resistance after multiple stretches, a hundred stretch-relaxation cycles test was applied to the electrode samples. Figure 2B showed the change of resistance at the relaxation point, and it could be found that after increasing 

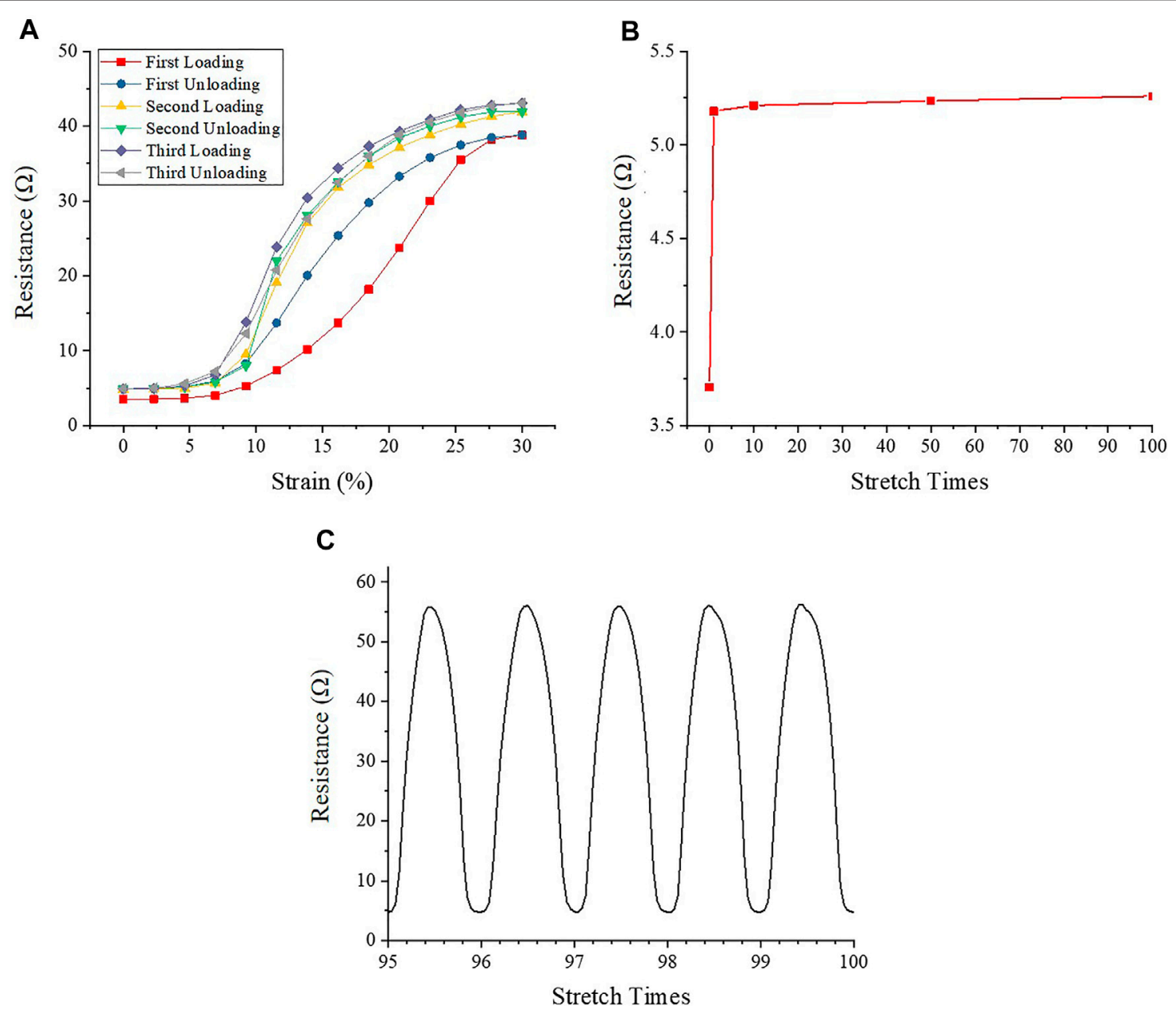

FIGURE 2 | The electrical resistance of AgNWs/PDMS electrode under mechanical loading cycles. (A) Electrical resistance during repeated stretch-relaxation cycles (the first to third cycles). (B) Electrical resistance of AgNWs/PDMS electrode with no strain after stretch. (C) Electrical resistance during repeated stretch-relaxation cycles (the last five cycles).

from 3.71 to $5.18 \Omega$ during the first three cycles, the resistance of the electrode samples was almost unchanged during the subsequent cycles. The resistance change in the last five stressrelaxation cycles was similar (Figure $\mathbf{2 C}$ ), and the average change of the maximum resistance was less than $0.05 \Omega$, which is negligible.

\section{Effects of Electrical Stimulation on PC12 Cell Growth}

The calcein AM stained PC12 cells were imaged at 24, 72, and $120 \mathrm{~h}$ (Figure 3A). More cells were observed on the electrode surface under electrical stimulation compared with the control group. This was further verified with the cell number measurement using Image $J$ (version 1.8.0, National Institutes of Health, United States). As shown in Figure 3B, the cell number on the control group without electrical stimulation was $(1.07 \pm 0.32) \times 10^{3}$ cells $/ \mathrm{cm}^{2},(4.60 \pm 1.25) \times 10^{3}$ cells $/$ $\mathrm{cm}^{2}$, and $(20.89 \pm 3.16) \times 10^{3}$ cells $/ \mathrm{cm}^{2}$ at 24,72 , and $120 \mathrm{~h}$, respectively. The cell number increased 4.3 times after $72 \mathrm{~h}$ and 4.5 times after $120 \mathrm{~h}$, while cells were still not able to cover the whole sample area (Figure 3A). The cell number increased significantly with the intensity of electrical stimulation at 24 and $72 \mathrm{~h}$ (Figure 3B). Under $60 \mathrm{mV}$ electrical stimulation, the cell number was $(1.21 \pm 0.22) \times 10^{3}$ cells $/ \mathrm{cm}^{2},(6.86 \pm 1.26) \times$ $10^{3}$ cells $/ \mathrm{cm}^{2}$ and $(27.14 \pm 5.72) \times 10^{3}$ cells $/ \mathrm{cm}^{2}$ at 24,72 , and $120 \mathrm{~h}$, respectively. Under $120 \mathrm{mV}$ electrical stimulation, the cell number was $(1.65 \pm 0.26) \times 10^{3}$ cells $/ \mathrm{cm}^{2},(9.84 \pm 2.22) \times$ $10^{3}$ cells $/ \mathrm{cm}^{2}$ and $(29.07 \pm 5.58) \times 103$ cells $/ \mathrm{cm}^{2}$ at 24,72 , and $120 \mathrm{~h}$, respectively. Under $240 \mathrm{mV}$ electrical stimulation, the cell number was $(1.97 \pm 0.33) \times 10^{3}$ cells $/ \mathrm{cm}^{2},(11.96 \pm 2.44) \times$ $10^{3}$ cells $/ \mathrm{cm}^{2}$ and $(30.68 \pm 5.71) \times 10^{3} \mathrm{cells} / \mathrm{cm}^{2}$ at 24,72 , and $120 \mathrm{~h}$, respectively. The cell number reached a similar level for all electrical stimulation samples at $120 \mathrm{~h}$ and cover the whole cell culture space. The cell number for the $240 \mathrm{mV}$ group increased 6.8 times, while the 60 and $120 \mathrm{mV}$ groups increased 5.6 times and 6 times, respectively, indicating that electrical stimulation can effectively promote the proliferation of PC12 cells, and this 

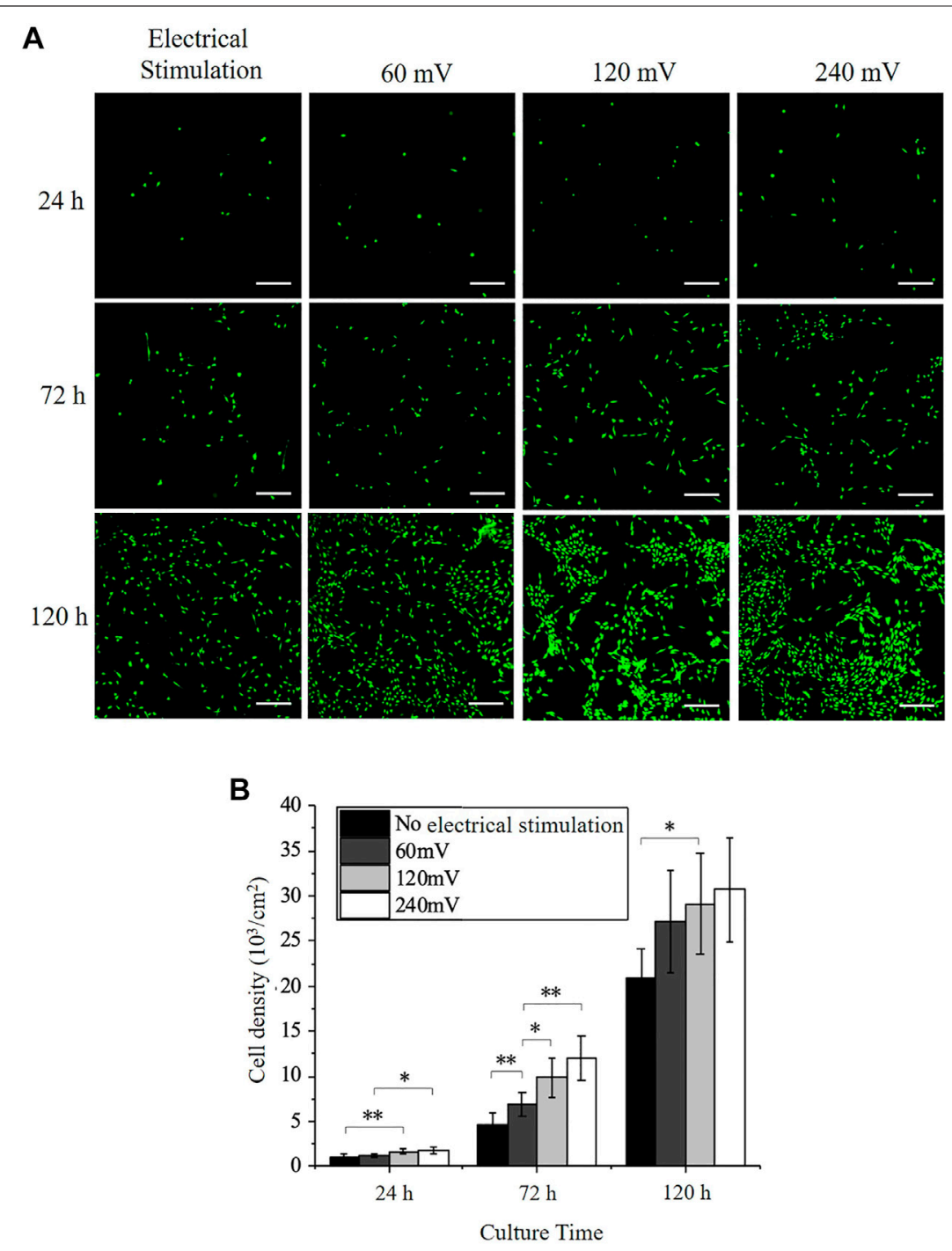

FIGURE 3|PC12 Immunofluorescence staining and cell proliferation under electrical stimulation. (A) Immunofluorescence images of PC12 cells cultured without or with electrical stimulation. Scale bar: $200 \mu \mathrm{m}$. (B) Cell densities of PC12 cultured on the electrodes. The significant difference between all the groups is indicated (*, $p<0.05 ; * *, p<0.01 ; n>10$ images).

effect became more pronounced as the intensity of electrical stimulation increased.

\section{Effects of Electrical Stimulation on PC12 Cell Axon Outgrowth}

The PC12 cell axon outgrowth was also analyzed and indicated with red dotted lines (Figure 4A). The length of the axon of the PC12 cells increased after from 24 to $120 \mathrm{~h}$ in all groups. The axon length of PC12 cells on control group at 24, 72, and $120 \mathrm{~h}$ were $55.81 \pm 18.79 \mu \mathrm{m}, 100.96 \pm 23.77 \mu \mathrm{m}$, and $129.67 \pm 30.46 \mu \mathrm{m}$, respectively. The axon length increased by $45 \mu \mathrm{m}$ from 24 to $72 \mathrm{~h}$ and only increased by $29 \mu \mathrm{m}$ from 72 to $120 \mathrm{~h}$, indicating that the outgrowth rate of the axon decreased with time in the control group. The axon length of PC12 cells under $60 \mathrm{mV}$ at 24, 72, and $120 \mathrm{~h}$ was $58.98 \pm 14.21 \mu \mathrm{m}, 111.15 \pm 30.54 \mu \mathrm{m}$, and $140.58 \pm$
$30.15 \mu \mathrm{m}$, respectively; the axon length of PC12 cells under $120 \mathrm{mV}$ at 24,72 , and $120 \mathrm{~h}$ was $63.55 \pm 15.76 \mu \mathrm{m}, 121.13 \pm$ $32.72 \mu \mathrm{m}$, and $142.04 \pm 30.38 \mu \mathrm{m}$, respectively; and the axon length of PC12 cells under $240 \mathrm{mV}$ at 24, 72, and $120 \mathrm{~h}$ was $70.01 \pm 14.77 \mu \mathrm{m}, 128.53 \pm 29.6 \mu \mathrm{m}$, and $141.18 \pm 27.86 \mu \mathrm{m}$, respectively. The axon outgrowth was slightly increased as the increases of electrical stimulation intensity at $24 \mathrm{~h}$, although significant differences were only found between the $120 \mathrm{mV}$ and the control group, and the $240 \mathrm{mV}$ and the $120 \mathrm{mV}$ group. The same phenomenon was also observed at $72 \mathrm{~h}$ with more significant differences observed, between the $60 \mathrm{mV}$ and the control group, the $120 \mathrm{mV}$ and the $60 \mathrm{mV}$, and the $240 \mathrm{mV}$ and the $60 \mathrm{mV}$. All the three electrical stimulation groups showed similar axon outgrowth after $120 \mathrm{~h}$, but slightly higher than the control group, indicating that electrical stimulation could promote axon outgrowth. 


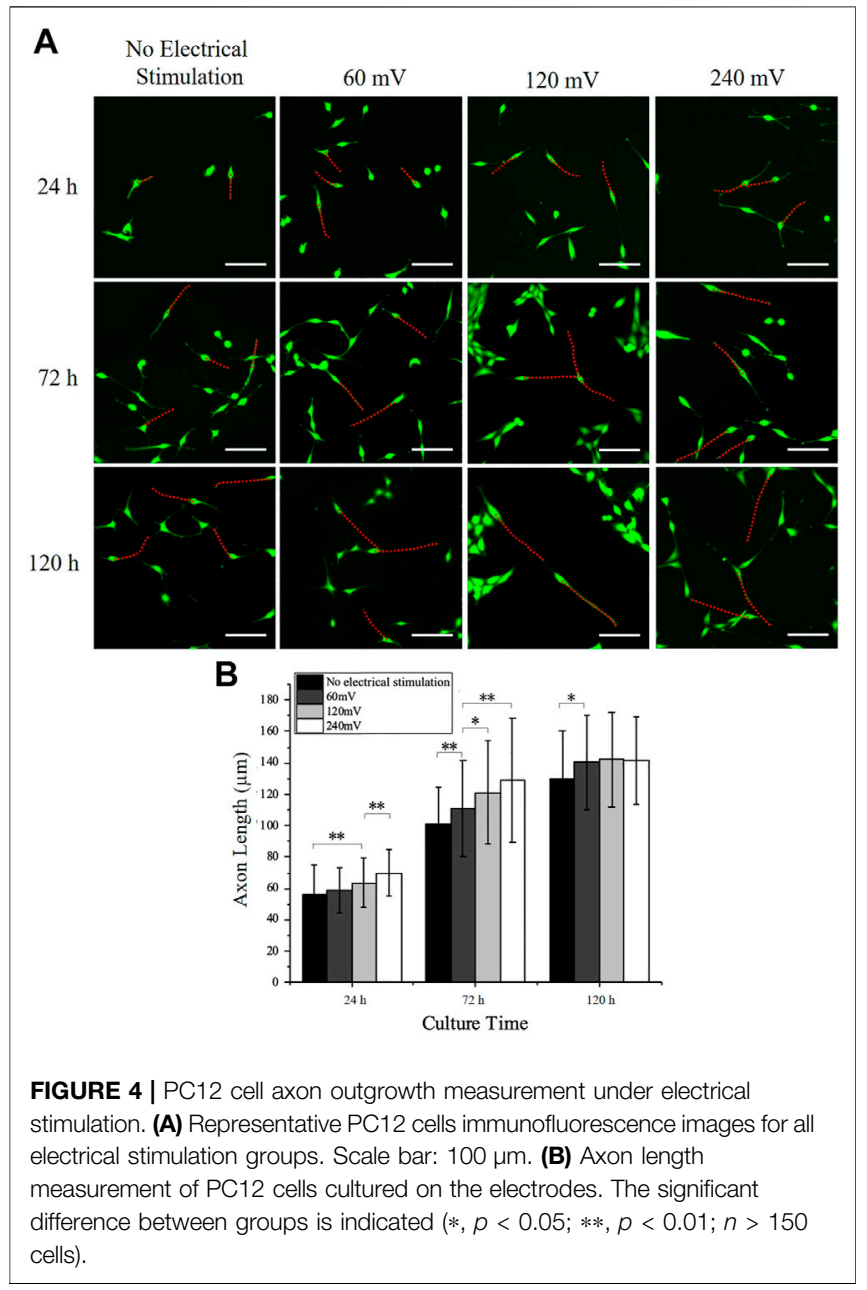

\section{Effects of the Electromechanical Stimulation on PC12 Cell Outgrowth}

PC12 cells were cultured on the electrodes under electromechanical stimulation to analyze the effects on the PC12 cell proliferation and axon outgrowth. Based on the electrical stimulation alone results (Effects of Electrical Stimulation on PC12 Cell Growth to Effects of Electrical Stimulation on PC12 Cell Axon Outgrowth), the electrical stimulation intensity of $240 \mathrm{mV}$ in combination with the mechanical stretching stimulation with a tensile strain of $10 \%$ and a frequency of $0.25 \mathrm{~Hz}$ was used as the electromechanical stimulation parameters. The PC12 cell densities (Figure 5) under electromechanical stimulation at 24,72 , and $120 \mathrm{~h}$ were $(1.23 \pm 0.24) \times 10^{3} \mathrm{cells} / \mathrm{cm}^{2}$, $(6.94 \pm 1.59) \times 10^{3} \mathrm{cells} / \mathrm{cm}^{2}$ and $(26.88 \pm 5.36) \times 10^{3}$ cells/ $\mathrm{cm}^{2}$, respectively. Compare with the control group (without any stimulation), PC12 cells under electromechanical stimulation had significantly larger cell numbers at 72 and $120 \mathrm{~h}$, indicating that the electromechanical stimulation can also promote cell proliferation. The PC12 cell densities under electromechanical stimulation at all time points were similar to the $60 \mathrm{mV}$ electrical stimulation group, while smaller than the $120 \mathrm{mV}$ electrical stimulation group (Figure 3). This may be attributed to the periodic change of the electrode resistance during stretching, resulting in larger equivalent resistance of the electrodes (Figure 2), and therefore larger voltage was needed to achieve the same effect as the $240 \mathrm{mV}$ samples.

The axon growth of the PC12 cells under the electromechanical stimulation was also analyzed (Figures 6A,B). The axon outgrowth of PC12 cells with electromechanical stimulation at 24,72 , and $120 \mathrm{~h}$ were $60.12 \pm 13.84 \mu \mathrm{m}, 116.01 \pm 22.55 \mu \mathrm{m}$, and $141.29 \pm$ $39.15 \mu \mathrm{m}$, respectively, which were significantly longer than the corresponding values of the control group. PC12 cells under electromechanical stimulation showed similar axon length as the $60 \mathrm{mV}$ electrical stimulation group but shorter than the $120 \mathrm{mV}$ electrical stimulation group, similar to the phenomenon observed for cell proliferation resulting from the larger equivalent resistance during stretching. The axon length reached $141.29 \pm 39.15 \mu \mathrm{m}$ after $120 \mathrm{~h}$ of culture, which was similar to the electrical stimulation alone group that the PC12 cells reached their maximum axon length.

The angle between the axon and stretch direction $(\theta)$ was further analyzed (Figure 6C). Interestingly, the axon of PC12 cells under electromechanical stimulation demonstrated a stretch direction-dependent growth, which was not observed in the control group and electrical stimulation alone groups. The percentages of cells that aligned perpendicularly $\left(60^{\circ} \leq \theta \leq\right.$ $120^{\circ}$ and $240^{\circ} \leq \theta \leq 300^{\circ}$ ) to the stretching direction were $65.45,79.72,84.59 \%$ at 24,72 , and $120 \mathrm{~h}$, respectively, indicating that the stretching stimulation influenced the preferred direction of the PC12 cell axon outgrowth.

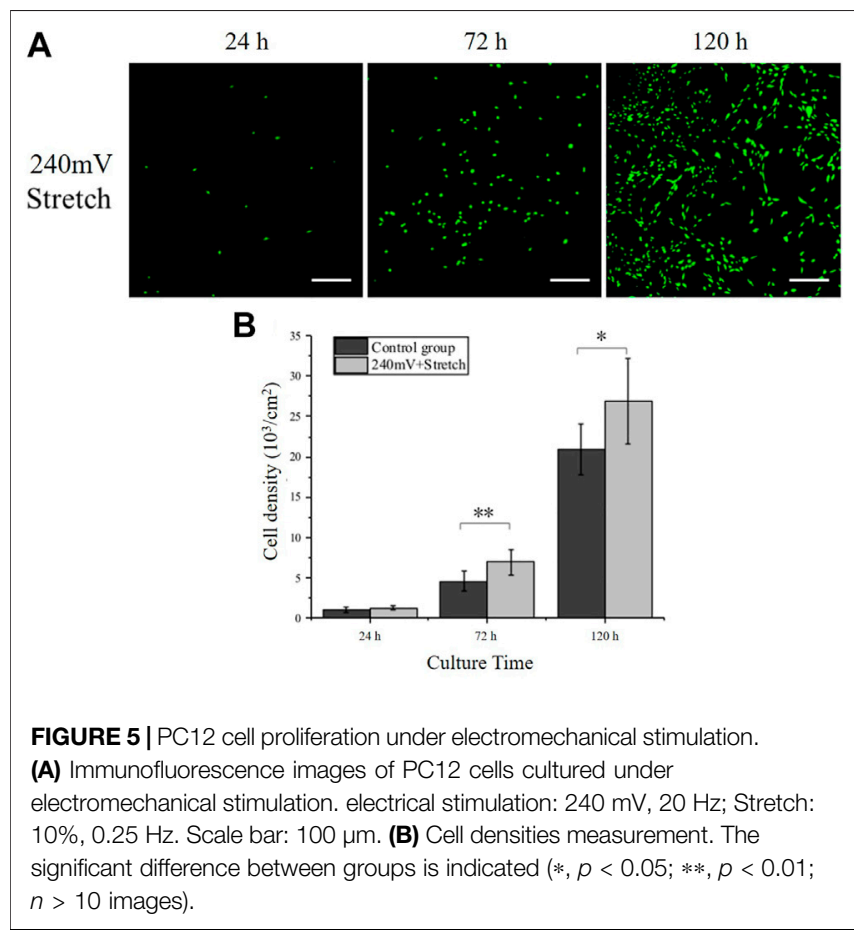




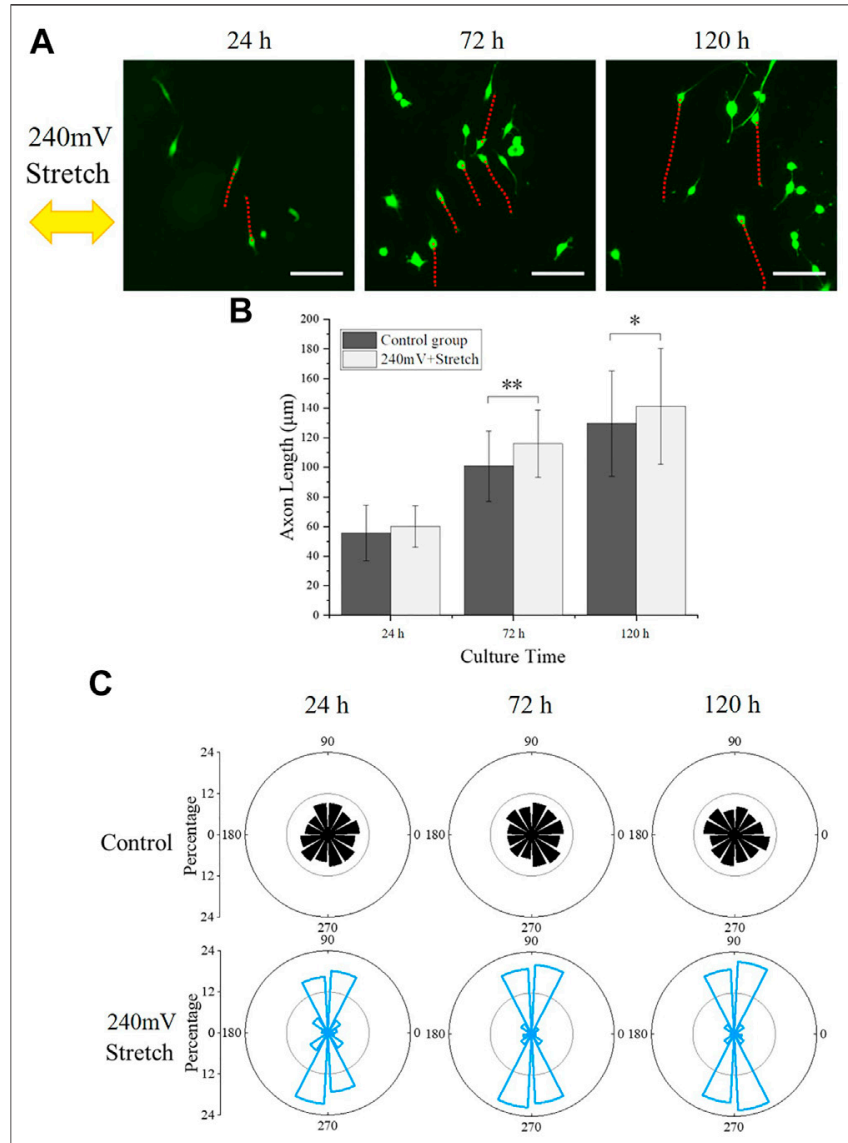

FIGURE 6 | PC12 cell axon orientation analysis. (A) PC12 cells

fluorescence images under electromechanical stimulation, with red dotted lines indicating the axon outgrowth and the yellow arrow representing the stretching direction. Scale bar: $100 \mu \mathrm{m}$. (B) Statistical results of axon length. The significant difference between groups is indicated $(*, p<0.05$; $* *, p<0.01 ; n>150$ cells). (C) Statistical angular distribution of axon orientation based on the fluorescence images.

\section{DISCUSSION}

In this study, AgNWs/PDMS electrode was developed to investigate the effects of electromechanical stimulation on PC12 cell proliferation and axon outgrowth. Although Agbased materials have inherently high electrical conductivity, their application as biomaterial was limited due to the possible releasement of toxic Ag oxidation and Ag ions (Hirai et al., 2016). In the present work, AgNWs were encapsulated into the PDMS model which prevented the release of toxic Ag ions and improved the electrode biocompatibility, while keeping the benefit of high electrical conductivity and establishment of a stable electric field. The use of the PDMS model not only prevented the leak of toxic byproducts but also provided sufficient mechanical strength and high elasticity to protect the AgNWs from breaking during stretching (Figure 2), which provided a promising platform to investigate the effects of electromechanical stimulation on PC12 cell proliferation and axon outgrowth.
The preceding results have clearly shown that electrical stimulation promotes the PC12 cell proliferation and axon outgrowth on AgNWs/PDMS electrodes. Similar results were found in previous studies: PC12 cells cultured on Polypyrrolecoated silk fibroin nanofibers (Sun et al., 2016) and P19 neurons (Hronik-Tupaj et al., 2013) cultured on silk film showed significantly higher cell proliferation rate and axon extension under electrical stimulation. Such phenomena could be explained by the effect of electrical stimulation on the gene expression and protein synthesis that regulate cell proliferation and axon outgrowth (Kimura et al., 1998). The myelin gene expression and neurotrophin secretion have been enhanced in nerve cells under electrical stimulation, which could lead to a longer axon outgrowth and a higher proliferation rate (Wu et al., 2016). Electrical stimulation was also found to upregulate the secretion of ciliary neurotrophic factor, glial cell-derived neurotrophic factor and fibroblast growth factor I, which could enhance the axon growth (Du et al., 2016). PC12 cellular membrane proteins, like F-actin and stress fibers, are critical factors that control the axon outgrowth, which are exposed to the mechanical stimulations first. When stretching amplitude and frequency are sufficiently large, cellular stress fibers are not able to maintain the axon orientations parallel to the cyclic stretch direction, and PC12 cells are forced to rotate to directions that are nearly perpendicular to the stretch direction (Lin et al., 2020). Another hypothesis is that cells will overexpress specific enzymes, like lysozyme, under mechanical stimulation, which could change the cellular protein expression, morphology, axon growth and direction (Kawasaki et al., 2009a; Kawasaki et al., 2009b; Ito et al., 2011). We plan to investigate the cell behavior mechanisms in our future studies. The cell number and axon outgrowth became similar for all groups after $120 \mathrm{~h}$, which could be caused by the limitation of cell culture space. When PC12 cells overgrow and axon interaction happens between cells, contact inhibition was triggered. Then, contact inhibition could cease the cell proliferation, division, and axon growth, and finally stop the cell from continuing growth.

To further reveal the effects of electromechanical stimulation on PC12 cell behavior, $240 \mathrm{mV}$ and $10 \%$ stretch strain at $0.25 \mathrm{~Hz}$ was applied. PC12 cells demonstrated enhanced proliferation and axon outgrowth, in comparison with the control group (Figures $5,6)$. However, both cell number and axon outgrowth at all time points were similar as $60 \mathrm{mV}$ electrical stimulation sample, while smaller than $120 \mathrm{mV}$ electrical stimulation sample. This could be due to the periodic change of electrode resistance during stretching, resulting in larger equivalent resistance of the electrode compared with the electrical stimulation alone group. Therefore, a larger voltage could be needed to overcome the electrical resistance change during the stretching and achieve a similar cell proliferation rate. Interestingly, the axon orientation of the PC12 cell was found to be more perpendicular to the stretch direction with electric stimulation and stretch. Embryonic rat cortical neurons obtained from pregnant rats cultured on PDMS also showed similar results, where the direction of neuronal outgrowth was perpendicular to the strain direction (Abraham et al., 2019). This perpendicular relation between axon outgrowth and stretch direction resembled the cyclic strain-induced actin 
reorientation, which could relate to the function of Src-family kinase activity that controls the cellular reorientation (Niediek et al., 2012). Overall, it was found that electric stimulation alone could enhance the PC12 cell proliferation and axon outgrowth on AgNWs/PDMS electrodes, while the electromechanical stimulation could offset the enhancement and direct the PC12 cell axon orientation.

\section{CONCLUSION}

In this work, PC12 cells were cultured on the AgNWs/PDMS electrodes to study the effects of electromechanical stimulation on cellular proliferation and axon outgrowth. The AgNWs/PDMS electrodes were developed and characterized to exhibit stable electrical resistance and good elasticity for stretching; therefore, an integrated electromechanical stimulation cell culture platform was established. The in vitro cell culture demonstrated that the prepared AgNWs/PDMS electrodes had good biocompatibility and could successfully support the adhesion and proliferation of the PC12 cells. The electric stimulation alone was found to enhance the proliferation rate and axon outgrowth of PC12 cells, and the cell number was increased with the increase of the electric stimulation intensity. However, the cell proliferation rate and axon outgrowth was dropped under electromechanical stimulation compared with the electric stimulation alone groups, while the axon orientation could be directed by the stretching direction. These results suggested that the AgNWs/PDMS electrodes provide a promising in vitro platform to investigate

\section{REFERENCES}

Abraham, J.-A., Linnartz, C., Dreissen, G., Springer, R., Blaschke, S., Rueger, M. A., et al. (2019). Directing Neuronal Outgrowth and Network Formation of Rat Cortical Neurons by Cyclic Substrate Stretch. Langmuir 35, 7423-7431. doi:10.1021/acs.langmuir.8b02003

Bhang, S. H., Jeong, S. I., Lee, T.-J., Jun, I., Lee, Y. B., Kim, B.-S., et al. (2012). Electroactive Electrospun Polyaniline/Poly[(L -Lactide)-Co- ( $\varepsilon$-caprolactone)] Fibers for Control of Neural Cell Function. Macromol. Biosci. 12, 402-411. doi:10.1002/mabi.201100333

Clarke, J. C., Tuft, B. W., Clinger, J. D., Levine, R., Figueroa, L. S., Allan Guymon, C., et al. (2011). Micropatterned Methacrylate Polymers Direct Spiral Ganglion Neurite and Schwann Cell Growth. Hearing Res. 278, 96-105. doi:10.1016/ j.heares.2011.05.004

Daly, W. T., Knight, A. M., Wang, H., de Boer, R., Giusti, G., Dadsetan, M., et al. (2013). Comparison and Characterization of Multiple Biomaterial Conduits for Peripheral Nerve Repair. Biomaterials 34, 8630-8639. doi:10.1016/ j.biomaterials.2013.07.086

Du, J., Chen, H., Qing, L., Yang, X., and Jia, X. (2018). Biomimetic Neural Scaffolds: a Crucial Step towards Optimal Peripheral Nerve Regeneration. Biomater. Sci. 6, 1299-1311. doi:10.1039/c8bm00260f

Du, Z., Bondarenko, O., Wang, D., Rouabhia, M., and Zhang, Z. (2016). Ex Vivo Assay of Electrical Stimulation to Rat Sciatic Nerves: Cell Behaviors and Growth Factor Expression. J. Cel. Physiol 231, 1301-1312. doi:10.1002/jcp.25230

Foley, J. D., Grunwald, E. W., Nealey, P. F., and Murphy, C. J. (2005). Cooperative Modulation of Neuritogenesis by PC12 Cells by Topography and Nerve Growth Factor. Biomaterials 26, 3639-3644. doi:10.1016/j.biomaterials.2004.09.048

Fozdar, D. Y., Lee, J. Y., Schmidt, C. E., and Chen, S. (2010). Hippocampal Neurons Respond Uniquely to Topographies of Various Sizes and Shapes. Biofabrication 2, 035005. doi:10.1088/1758-5082/2/3/035005 the sensing mechanisms of nerve cell behaviors to electromechanical cues, and can be potentially used as nerve conduit materials in the future.

\section{DATA AVAILABILITY STATEMENT}

The original contributions presented in the study are included in the article/Supplementary Material, further inquiries can be directed to the corresponding authors.

\section{AUTHOR CONTRIBUTIONS}

KLX and XXL analyzed the data and drafted the manuscript. XKL prepared the PDMS/AgNWs electrodes and performed in vitro cell culture. PW and JY revised and organized the experiments. JQ helped to revise the manuscript, and co-ordinate the study. JS is the principal investigator, designed the experiments, organize and revise the manuscript.

\section{FUNDING}

This work was supported by the National Natural Science Foundation of China (Grants 12125205, 91748209 and 52075482) and the Fundamental Research Funds for Central Universities of China (Grant 2020XZZX005-02).

Grove, M., and Brophy, P. J. (2014). FAK Is Required for Schwann Cell Spreading on Immature Basal Lamina to Coordinate the Radial Sorting of Peripheral Axons with Myelination. J. Neurosci. 34, 13422-13434. doi:10.1523/ jneurosci.1764-14.2014

Gu, X., Ding, F., Yang, Y., and Liu, J. (2011). Construction of Tissue Engineered Nerve Grafts and Their Application in Peripheral Nerve Regeneration. Prog. Neurobiol. 93, 204-230. doi:10.1016/j.pneurobio.2010.11.002

Gupta, R., Nassiri, N., Hazel, A., Bathen, M., and Mozaffar, T. (2012). Chronic Nerve Compression Alters Schwann Cell Myelin Architecture in a Murine Model. Muscle Nerve 45, 231-241. doi:10.1002/mus.22276

Han, Q., Chen, Y., Song, W., Zhang, M., Wang, S., Ren, P., et al. (2019). Fabrication of Agarose Hydrogel with Patterned Silver Nanowires for Motion Sensor. Biodes. Manuf. 2, 269-277. doi:10.1007/s42242-019-00051-w

Hirai, T., Yoshioka, Y., Izumi, N., Ichihashi, K.-i., Handa, T., Nishijima, N., et al. (2016). Metal Nanoparticles in the Presence of Lipopolysaccharides Trigger the Onset of Metal Allergy in Mice. Nat. Nanotech 11, 808-816. doi:10.1038/ nnano. 2016.88

Hoffman-Kim, D., Mitchel, J. A., and Bellamkonda, R. V. (2010). Topography, Cell Response, and Nerve Regeneration. Annu. Rev. Biomed. Eng. 12, 203-231. doi:10.1146/annurev-bioeng-070909-105351

Hronik-Tupaj, M., Raja, W. K., Tang-Schomer, M., Omenetto, F. G., and Kaplan, D. L. (2013). Neural Responses to Electrical Stimulation on Patterned Silk Films. J. Biomed. Mater. Res. 101A, 2559-2572. doi:10.1002/jbm.a.34565

Huang, Y.-C., and Huang, Y.-Y. (2006). Tissue Engineering for Nerve Repair. Biomed. Eng. Appl. Basis Commun. 18, 100-110. doi:10.4015/ s101623720600018x

Ishibashi, Y., Uesugi, K., and Morishima, K. (2016). Effect of Mechanical Stimulation on Neurite Outgrowth of Dorsal Root Ganglion Neurons toward Integrative Mechanobiologic Nerve Bridge. in International Symposium on System Integration SII, Shiroishi, Japan, December 13, 797-802. doi:10.1109/sii.2016.7844097 
Ito, Y., Kimura, T., Nam, K., Katoh, A., Masuzawa, T., and Kishida, A. (2011). Effects of Vibration on Differentiation of Cultured PC12 Cells. Biotechnol. Bioeng. 108 (3), 592-599. doi:10.1002/bit.22961

Jahromi, M., Razavi, S., and Bakhtiari, A. (2019). The Advances in Nerve Tissue Engineering: From Fabrication of Nerve Conduit Toin Vivonerve Regeneration Assays. J. Tissue Eng. Regen. Med. 13, 2077-2100. doi:10.1002/term.2945

Jeong, J., Chou, N., and Kim, S. (2016). Long-term Characterization of Neural Electrodes Based on Parylene-Caulked Polydimethylsiloxane Substrate. Biomed. Microdevices 18 (3), 42-51. doi:10.1007/s10544-016-0065-Z

Kawasaki, T., Toyoda, M., Hoshino, Y., and Okahata, Y. (2009). Pulsed Ultrasound Effect on DNA Polymerase Reaction Monitored on a QCM. Chem. Lett. 38 (6), 538-539. doi:10.1246/cl.2009.538

Kawasaki, T., Toyoda, M., and Okahata, Y. (2009). Pulse Frequency-dependent Regulation of Lysozyme Reactivity under Pulsed Ultrasound Irradiation. Chem. Lett. 38 (6), 536-537. doi:10.1246/cl.2009.536

Kimura, K., Yanagida, Y., Haruyama, T., Kobatake, E., and Aizawa, M. (1998). Gene Expression in the Electrically Stimulated Differentiation of PC12 Cells. J. Biotechnol. 63, 55-65. doi:10.1016/s0168-1656(98)00075-3

Kotwal, A., and Schmidt, C. E. (2001). Electrical Stimulation Alters Protein Adsorption and Nerve Cell Interactions with Electrically Conducting Biomaterials. Biomaterials 22, 1055-1064. doi:10.1016/s0142-9612(00)00344-6

Krishnamoorthy, S., Zhang, Z., and Xu, C. (2020). Guided Cell Migration on a Graded Micropillar Substrate. Bio-des. Manuf. 3, 60-70. doi:10.1007/s42242020-00059-7

Lin, J., Li, X., Yin, J., and Qian, J. (2020). Effect of Cyclic Stretch on Neuron Reorientation and Axon Outgrowth. Front. Bioeng. Biotechnol. 8, 597867. doi: $10.3389 /$ fbioe. 2020.597867

Liu, J., Zhang, B., Li, L., Yin, J., and Fu, J. (2021). Additive-lathe 3D Bioprinting of Bilayered Nerve Conduits Incorporated with Supportive Cells. Bioactive Mater. 6, 219-229. doi:10.1016/j.bioactmat.2020.08.010

Love, J. M., Bober, B. G., Orozco, E., White, A. T., Bremner, S. N., Lovering, R. M., et al. (2017). mTOR Regulates Peripheral Nerve Response to Tensile Strain. J. Neurophysiol. 117, 2075-2084. doi:10.1152/jn.00257.2016

Lu, M.-C., Ho, C.-Y., Hsu, S.-F., Lee, H.-C., Lin, J.-H., Yao, C.-H., et al. (2008). Effects of Electrical Stimulation at Different Frequencies on Regeneration of Transected Peripheral Nerve. Neurorehabil. Neural Repair 22, 367-373. doi:10.1177/1545968307313507

Niediek, V., Born, S., Hampe, N., Kirchgessner, N., Merkel, R., and Hoffmann, B. (2012). Cyclic Stretch Induces Reorientation of Cells in a Src Family Kinaseand p130Cas-dependent Manner. Eur. J. Cel Biol. 91, 118-128. doi:10.1016/ j.ejcb.2011.10.003

Phillips, J. B., Smit, X., Zoysa, N. D., Afoke, A., and Brown, R. A. (2004). Peripheral Nerves in the Rat Exhibit Localized Heterogeneity of Tensile Properties during Limb Movement. J. Physiol. 557, 879-887. doi:10.1113/jphysiol.2004.061804

Shineh, G., Kordestani, S. S., Tahriri, M., and Tayebi, L. (2019). Evaluation of L929 Cell Morphology on Anthocyanin-Containing Gelatin-Based Hydrogel for Early Detection of Infection. Bio-des. Manuf. 2, 181-186. doi:10.1007/ s42242-019-00047-6

Sun, B., Wu, T., Wang, J., Li, D., Wang, J., Gao, Q., et al. (2016). Polypyrrole-coated Poly(l-Lactic Acid-Co- $\varepsilon$-Caprolactone)/silk Fibroin Nanofibrous Membranes
Promoting Neural Cell Proliferation and Differentiation with Electrical Stimulation. J. Mater. Chem. B 4, 6670-6679. doi:10.1039/c6tb01710j

Suo, H., Wang, Z., Dai, G., Fu, J., Yin, J., and Chang, L. (2018). Polyacrylonitrile Nerve Conduits with Inner Longitudinal Grooved Textures to Enhance Neuron Directional Outgrowth. J. Microelectromech. Syst. 27, 457-463. doi:10.1109/ jmems.2018.2810097

Tang, S., Zhu, J., Xu, Y., Xiang, A. P., Jiang, M. H., and Quan, D. (2013). The Effects of Gradients of Nerve Growth Factor Immobilized PCLA Scaffolds on Neurite Outgrowth In Vitro and Peripheral Nerve Regeneration in Rats. Biomaterials 34, 7086-7096. doi:10.1016/j.biomaterials.2013.05.080

Wang, Z., Wu, Y., Xiang, Y., Kruth, M. B., Wei, P., Dai, G., et al. (2021). Efficacy of Large Groove Texture on Rat Sciatic Nerve Regeneration In Vivo Using Polyacrylonitrile Nerve Conduits. Ann. Biomed. Eng. 49, 394-406. doi:10.1007/s10439-020-02560-7

Wieringa, P. A., Gonçalves de Pinho, A. R., Micera, S., van Wezel, R. J. A., and Moroni, L. (2018). Biomimetic Architectures for Peripheral Nerve Repair: A Review of Biofabrication Strategies. Adv. Healthc. Mater. 7, e1701164. doi:10.1002/adhm.201701164

Wu, Y., Xiang, Y., Fang, J., Li, X., Lin, Z., Dai, G., et al. (2019). The Influence of the Stiffness of GelMA Substrate on the Outgrowth of PC12 Cells. Biosci. Rep. 39, BSR20181748. doi:10.1042/BSR20181748

Wu, Y., Wang, L., Guo, B., Shao, Y., and Ma, P. X. (2016). Electroactive Biodegradable Polyurethane Significantly Enhanced Schwann Cells Myelin Gene Expression and Neurotrophin Secretion for Peripheral Nerve Tissue Engineering. Biomaterials 87, 18-31. doi:10.1016/j.biomaterials.2016.02.010

Yin, J., Wang, Z., Chai, W., Dai, G., Suo, H., Zhang, N., et al. (2017). Fabrication of Inner Grooved Hollow Fiber Membranes Using Microstructured Spinneret for Nerve Regeneration. J. Manufacturing Sci. Eng. 139, 111007. doi:10.1115/1.4037430

Zhang, D., Suo, H., Qian, J., Yin, J., Fu, J., and Huang, Y. (2020). Physical Understanding of Axonal Growth Patterns on Grooved Substrates: Groove ridge Crossing versus Longitudinal Alignment. Bio-des. Manuf. 3, 348-360. doi:10.1007/s42242-020-00089-1

Conflict of Interest: The authors declare that the research was conducted in the absence of any commercial or financial relationships that could be construed as a potential conflict of interest.

Publisher's Note: All claims expressed in this article are solely those of the authors and do not necessarily represent those of their affiliated organizations, or those of the publisher, the editors and the reviewers. Any product that may be evaluated in this article, or claim that may be made by its manufacturer, is not guaranteed or endorsed by the publisher.

Copyright (c) $2021 \mathrm{Xu}, \mathrm{Liu}, \mathrm{Li}$, Yin, Wei, Qian and Sun. This is an open-access article distributed under the terms of the Creative Commons Attribution License (CC BY). The use, distribution or reproduction in other forums is permitted, provided the original author(s) and the copyright owner(s) are credited and that the original publication in this journal is cited, in accordance with accepted academic practice. No use, distribution or reproduction is permitted which does not comply with these terms. 